\title{
Consideration of a Neuromotor Concussion Subtype
}

Mini Review
Volume 2 Issue $2-2021$
Author Details
Mark Williams ${ }^{1}$, Blair Pickett ${ }^{2}$, Julian Keith ${ }^{3}$, Len Lecci ${ }^{3 *}$
${ }^{1}$ Department of Medicine, University of North Carolina Chapel Hill School of Medicine and New Hanover Regional Medical Center, USA
${ }^{2}$ Research Associate, USA
${ }^{3}$ Department of Psychology, University of North Carolina Wilmington, Wilmington, NC, USA
*Corresponding author
Dr. Len Lecci, Department of Psychology, University of North Carolina Wilmington, 601 South College Rd, Wilmington, NC,
28403-5612, USA
Article History
Received: June 04, $2021 \quad$ Accepted: June 24, $2021 \quad$ Published: June 29, 2021

\begin{abstract}
A recent Consensus Conference proposed subtyping concussive injuries into 5 categories. We propose adding a neuromotor subtype. Abnormal motion is a key feature of brain injury, as concussions can impact the neurological systems controlling gait. Neuromotor function can remain abnormal after symptom resolution and may be superior to self-report for tracking recovery. Neuromotor function can also define co-occurring orthopedic injuries and reveal vulnerabilities which could reduce injury risk.
\end{abstract}

Keywords: Concussion; Gait assessment; Head injury; Neuromotor subtype; Traumatic brain injury

\section{Mini Review}

In 2016, a Consensus Conference provided guidelines for the evaluation of patients experiencing a potential concussion [1] from which a framework to define concussion subtypes, and concussionassociated conditions was derived [2]. Based on a metanalysis, five concussion subtypes were identified: Cognitive, ocular-motor, headache/migraine, vestibular, and anxiety-mood [2]. Each subtype includes a defined prevalence and the measurements used for analysis. These consensus guidelines are a significant advancement that can aid in identifying, evaluating, and following patients with suspected brain injury. We propose that a neuromotor, gait-specific subtype could be an important addition to this nosology, and could result in clinicians and researchers better articulating and assessing the neuromotor consequences of concussion.

There are several justifications for considering a gait concussion subtype and conducting a formalized assessment of gait. First, a sizable portion of the brain is involved in supporting motion. Second, gait abnormalities have been identified as an acute symptom of concussion $[3,4]$. Third, because concussions can be caused by a wide range of events including athletic and recreational injuries, motor vehicle and bicycle crashes, falls, altercations, intentional self-harm, and workrelated accidents $[5,6]$, the sequalae of such events may be equally heterogenous. In fact, concussions can include sensory symptoms such as numbness, dizziness, or instability, and the neuromotor consequences of these symptoms would help to more fully capture the extent of the injury, localize the site of injury, and inform recovery [7]. Moreover, neurological patterns of gait can capture neuromuscular, orthopedic, and biomechanical complications that can co-occur with concussion [8]. For example, gait asymmetries, variability, shuffling, apraxia, or unsteadiness represent some of these gait characteristics, though they may be extremely subtle and invisible to an inexpert observer.

Another advantage of including a formal gait evaluation is that it provides information separate from the patient's self-report. In contrast, the concussion framework forwarded in 2016 relies heavily on self-reports, with headache/migraine and anxiety-mood subtypes being solely based on self-reported symptoms. Consequently, the objective evaluation of neuromotor function could complement selfreported symptoms, and is especially relevant for those with difficulty communicating their symptoms (e.g., children, dementia patients) or those trying to manipulate the evaluation (e.g., personal injury claimants who are malingering, or athletes eager to return to sport) [9]. Because locomotion is an automatic, unconscious activity [10], the automaticity of walking and balance while walking are often implicated in concussions, such that these automatic and unconscious activities may require conscious awareness and effort to function normally [10]. Because gait is mainly unconscious, patients may be less aware of gait-related dysfunction or the extent to which conscious control over gait is being exerted due to declines in gait automaticity. 
Although there are some exceptions (e.g., NIH 4-Meter gait test, The Up and Go test, the Performance-oriented Mobility Assessment), motion-based assessments are often communicated as a subjective clinical impression made by an expert observer [11-16]. The challenges with these subjective impressions include scarcity of expert observers, limited inter-rater reliability, and difficulty in communicating subtle observations. An objective analysis of human motion can generate reproducible indicators with greater measurement sensitivity. Thus, systematic analysis of gait using, for example, wearable motion sensors can provide objective, noninvasive, and unobtrusive data for longitudinal tracking of an athlete's neuromotor functioning from baseline through recovery. This analysis could allow for the direct, quantitative comparison between the magnitude of concussive or subconcussive dysfunction both relative to baseline data and relative to population-based norms. Identifying characteristic deviations from these norms, particularly in the context of sub-concussive injuries, could provide an early warning of functional change before disabilities become permanent. This is especially relevant given that multiple sub-concussive events may predispose one to significant future brain injury [17].

When considering injury prevention, it's important to note that numerous factors can underlie the risk of experiencing an injury, and this can include proper gait form and degree of effort or fatigue [18]. Thus, poor gait technique during exertion can amplify underlying vulnerabilities in our anatomy and posture. Rehabilitation of poor gait technique is essential after a concussive injury because subliminal compensatory patterns may produce poor habits and increase the likelihood of secondary injury. A gait evaluation can identify the proper technique, compensatory patterns, and maladaptive habits for such variables as gait symmetry, speed, variability, and energy expenditure. With this gait profile, athletic trainers and physical therapists can tailor a treatment program to reduce the risk of future injury. Moreover, subtle changes can be quantified and tracked to illustrate progress and areas needing further or alternative interventions.

In summary, concussions are heterogeneous injuries and a neuromotor (gait) subtype is an essential feature missing from the current nosology of concussion. Its inclusion as a concussion subtype could improve screening, diagnosis, treatment, and injury prevention.

\section{References}

1. Collins M, Kontos A, Okonkwo D, Almquist J, Bailes J, et al. (2016) Statements of agreement from the targeted evaluation and active management (TEAM) approaches to treating concussion meeting held in pittsburgh, October 15-16, 2015. Neurosurgery 79(6): 912-929.

2. Lumba Brown A, Teramoto M, Bloom OJ, Brody D, Chesnutt J, et al. (2020) Concussion guidelines step 2: evidence for subtype classification. Neurosurgery $86(1): 2-13$

3. Cavanaugh JT, Guskiewicz KM, Giuliani C, Marshall S, Mercer V, et al. (2005) Detecting altered postural control after cerebral concussion in athletes with normal postural stability. Br J Sports Med 39(11): 805-811.
4. Williams G, Morris M, Schache A, McCrory P (2009) Incidence of gait abnormalities after traumatic brain injury. Arch Phys Med Rehabil 90(4): 587-593.

5. Laker SR (2011) Epidemiology of concussion and mild traumatic brain injury. PM\&R 3(10): S354-S358.

6. Centers for Disease Control and Prevention (2019). Surveillance Report of Traumatic Brain Injury-related Emergency Department Visits, Hospitalizations, and Deaths-United States, 2014. Centers for Disease Control and Prevention, U.S. Department of Health and Human Services.

7. Lau BC, Kontos AP, Collins MW, Mucha A, Lovell MR (2011) Which on-field signs/symptoms predict protracted recovery from sport-related concussion among high school football players? Am J Sports Med 39(11): 2311-2318.

8. Astephen Wilson JL, Deluzio KJ, Dunbar MJ, Caldwell GE, HubleyKozey CL (2011) The association between knee joint biomechanics and neuromuscular control and moderate knee osteoarthritis radiographic and pain severity. Osteoarthritis Cartilage 19(2): 186-193.

9. Silver JM (2012) Effort, exaggeration and malingering after concussion. J Neurol Neurosurg Psychiatry 83(8): 836-841

10. Allali G, Montembeault M, Griffa A, Beauchet O (2020) Default mode network and the timed up and go in MCI: a structural covariance analysis. Exp Gerontol 129: 110748.

11. Kallen M, Slotkin J, Griffith J (2012) Toolbox 4-Meter Walk Gait Speed Test Technical Manual 14-15.

12. Podsiadlo D, Richardson S (1991) The timed "up \& go": a test of basic functional mobility for frail elderly persons. J Am Geriatr Soc 39(2): 142-148.

13. Tinetti ME, Williams TF, Mayewski R (1986) Fall risk index for elderly patients based on number of chronic disabilities. Am J Med 80(3): 429434 .

14. Santo A, Lynall RC, Guskiewicz KM, Mihalik JP (2017) Clinical utility of the sport concussion assessment tool $_{3}\left(\mathrm{SCAT}_{3}\right)$ tandem-gait test in high school athletes. J Athl Train 52(12): 1096-1100.

15. Schneiders AG, Sullivan SJ, Kvarnström J, Olsson M, Ydén T, et al. (2010) The effect of footwear and sports-surface on dynamic neurological screening for sport-related concussion. J Sci Med Sport 13(4): 382-386.

16. Howell DR, Lynall RC, Buckley TA, Herman DC (2018) Neuromuscular control deficits and the risk of subsequent injury after a concussion: a scoping review. Sports Med 48(5): 1097-1115.

17. Marchi N, Bazarian JJ, Puvenna V, Janigro M, Ghosh C, et al. (2013) Consequences of repeated blood-brain barrier disruption in football players. PloS One 8(3): e56805.

18. Benjaminse A, Habu A, Sell TC, Abt J P, Fu F H, et al. (2008) Fatigue alters lower extremity kinematics during a single-leg stop-jump task. Knee Surg Sports Traumatol Arthrosc. 16(4): 400-407. 